\title{
ExoPranayama: a biofeedback-driven actuated environment for supporting yoga breathing practices
}

\author{
Stuart Moran $^{1} \cdot$ Nils Jäger $^{1} \cdot$ Holger Schnädelbach ${ }^{1} \cdot$ Kevin $_{\text {Glover }}{ }^{1}$
}

Received: 29 July 2015/ Accepted: 4 March 2016/Published online: 5 April 2016

(c) The Author(s) 2016. This article is published with open access at Springerlink.com

\begin{abstract}
Both breathing and internal self-awareness are an integral part of any yoga practice. We describe and discuss the development of ExoPranayama, an actuated environment that physically manifests users' breathing in yoga. Through a series of trials with yoga practitioners and expert teachers, we explore its role in the practice of yoga. Our interview results reveal that biofeedback through the environment supported teaching and improved selfawareness, but it impacted group cohesion. Two practical uses of the technology emerged for supporting breath control in yoga: (1) biofeedback can provide new information about users' current internal states; (2) machinedriven feedback provides users with a future state or goal and leads to improved cohesiveness.
\end{abstract}

Keywords Adaptive environment - Breathing ·

ExoBuilding $\cdot$ Physiology $\cdot$ Pranayama $\cdot$ Teaching $\cdot$ Yoga

\section{Introduction}

The ability to breathe is an essential function for sustaining our lives. It is a subconscious activity controlled by our autonomic nervous system that we are routinely unaware of. However, it is possible for us to override this system in order to moderate our breathing, and there are a number of meditation disciplines and relaxation therapies, which focus on breath control. Yoga is a discipline which seeks to achieve health and relaxation through a combination of

Holger Schnädelbach

holger.schnadelbach@nottingham.ac.uk

1 Mixed Reality Lab, School of Computer Science, University of Nottingham, Jubilee Campus, Nottingham NG81BB, UK dynamic postures and Pranayama, the practice of breath (prana) regulation (ayama). To regulate breathing effectively, a person must be acutely aware of their body's internal processes. They can then control their heart rate and respiration by observing their body's performance, a process reminiscent of, and preceding, biofeedback [1]. Also, physical props are often used to support bodily alignment and augment this internal sensing process, highlighting an intriguing interplay between physical and cognitive activities in breath regulation.

In this paper, we seek to explore how biofeedback-based physical actuations might be embedded into, and support, these established practices. We build on an existing prototype called ExoBuilding [2] that demonstrates how physiological data, such as respiration, can be used to actuate an environment. We use ExoBuilding as our starting point as, on the surface, it shares a focus on respiration with the practice of yoga. We inform the redesign of ExoBuilding for yoga in two specific ways: (1) "in-thewild" observations of a yoga class, providing an in-depth understanding of the practice, and (2) an exploratory iterative development cycle, which helped refine our designs. This led to the development of "ExoPranayama", a bespoke instance of ExoBuilding.

Our testing of ExoPranayama revealed an improvement in self-awareness and new insights for teachers about their students. This was at the cost of group cohesion, which was alleviated when biofeedback within the group was visually aggregated. ExoPranayama also surfaced a number of social concerns related to exposure of the invisible "innerself": competitiveness and deviation from the spiritual aspects of the practice. From these findings, we propose two practical uses of ExoPranayama for supporting breathing in yoga, focusing on the different outcomes of biofeedback and machine-regulated actuations. This work 
contributes to the understanding and use of physiology as part of a broader interactive group experience, with a particular emphasis on breathing. We also seek to address the current emphasis on measurement and quantification within biofeedback by exploring how physiological data may find a role in technology-supported spiritual practices [3]. In light of this, we offer a series of considerations for developers of biofeedback applications for yoga and other physiological-related practices.

\section{Related work}

In its broadest sense, technology is already widely used for practising yoga in the form of props such as straps and blocks. These props have been described as mediators with the aim of providing physical feedback, creating awareness and connections between parts of the body [13]. More modern computer-based technologies have typically sought to emulate this same type of feedback, albeit in a more efficient and/or novel medium. For example, commercial technology such as the Nintendo Wii-Balance board uses pressure sensors to support and teach breathing [4]. Microsoft's Kinect makes use of computer vision techniques to track users' limbs in real time, providing visual and auditory feedback about their posture. Kinect has also been used to assist the partially sighted and blind practice yoga [5]. "Yoga Social Mats" [6] support a more social experience by sharing sensed activity through tablets. The limitations of these technologies are that they focus on collecting visible data on the "outside" of the body, rather than capturing and presenting the physiology of the individual which resides inside [7].

One of the goals of a yoga practice, however, is to look inward and control internal mechanisms such as respiration and heart rate, ultimately leading to inner peacefulness and resilience. We suggest that biofeedback technology might be used to support this by externalizing invisible human physiological processes. Wearable devices such as "Basis" (www.mybasis.com/) can be used in a yoga practice for measuring heart rate. Sensors for measuring respiration have also been embedded in clothing for a more natural experience [8]. Much of the above technology has been used in immersive systems such as the meditation chamber [9] where respiration manipulates visuals and sounds. Similarly, Sonic Cradle [10] is a sensory deprivation chamber designed to support mindfulness, where respiration creates real-time soundscapes.

Our primary focus in this research is to explore biofeedback-based physical actuations, of which there has been limited work. MoodWings [11] is a wearable device in the shape of a butterfly, with wings that actuate in real time to a user's heart rate. This type of interaction has been shown to be more effective at reducing stress than traditional approaches found in meditation [12], thus showing promise for actuated technology in yoga practices. "Broncomatic" is an actuated amusement ride controlled by the riders' breath [13]. The system was used to explore breathing as a control mechanism, revealing it to be a rich mode of interaction. Finally, ExoBuilding [2] is a tent-like structure that changes its height, volume and shape based on its inhabitants' real-time physiological data. Designed to investigate the relationship between people and buildings, it has been shown to facilitate regular respiratory patterns [2]. As shown above, biofeedback technologies have been introduced to and used in yoga practice, but we are not aware of such technology having ever been used in a group context.

\section{Design and development}

Our starting point was the existing ExoBuilding prototype and its ability to capture physiological data, representing the unobservable "inner space" of a person, and relaying that information in the form of physical actuations of the environment. ExoBuilding consists of jersey fabric stretched over an aluminium spine, which itself is attached to two ceiling-mounted servomotors. The combination of physical structure, biosensing technology (MindMedia NeXus10 and BioTrace + www.mindmedia.info) and a middleware platform [14] allows direct physiological interaction with the environment. The servomotors receive physiological data from a respiration belt sensor, which (by stretching) measures the rise/fall of a person's abdomen in accordance with their breath. The motors can also be driven by a machine-generated signal, creating two means of sourcing data: biofeedback and machine-driven. This translates into a change (up to $30 \mathrm{~cm}$ ) in the position of the main spine (Fig. 1), altering ExoBuilding's shape by stretching the fabric.

To explore ExoBuilding's applicability in a (multi-participant) yoga practice, we pursued a user-focused iterative development approach. Working with end users allowed us to embed their needs in co-developed iterative prototypes [15]. Yoga teachers and students are likely to have different experiences within a practice, and as such, we sought to gain insight from both perspectives to inform the redesign of ExoBuilding. Figure 2 shows an overview of the design process, separated into phases. Each of the phases focuses on different user groups and prototype iterations.

We first sought to better understand the practice of yoga by observing a series of classes (P1). We then interviewed a group of professional yoga teachers (P2) and followed this with a trial involving a large yoga class (P3). Then we proceeded to focus more closely on a pair of yoga experts 

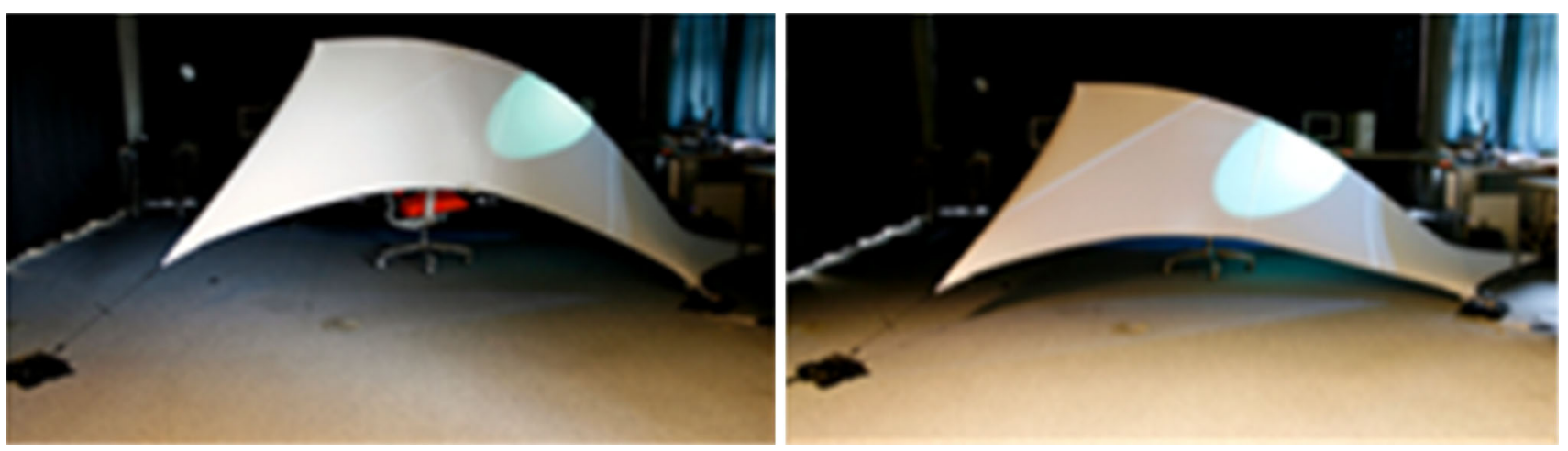

Fig. 1 ExoBuilding [2] in its highest and lowest states

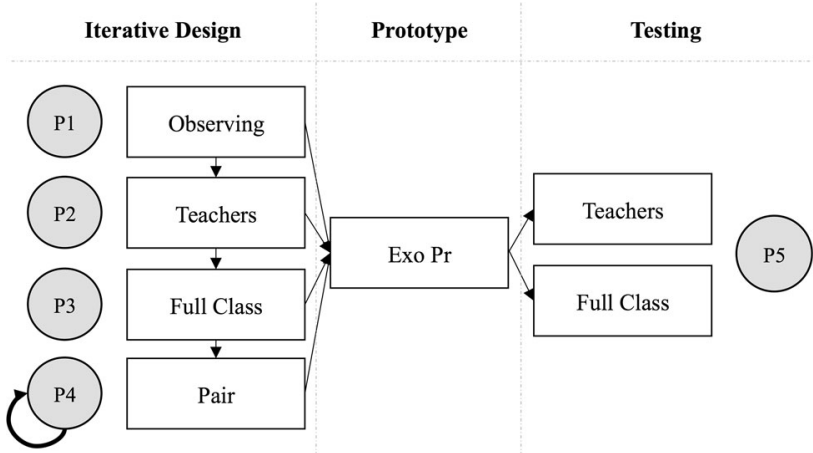

observations, we supplement the first-hand experience with relevant interview findings from phase 2, which saw a series of in-depth interviews with five practising yoga teachers (T1-T5) who run classes locally. We begin with a brief outline of our (now practice informed) understanding of yoga, followed by details of key observations relevant to the design of ExoBuilding.

\subsection{Defining yoga}

Fig. 2 Overview of the phases of the design process

(P4), with a series of iterative trials leading to direct changes to ExoBuilding. The combined results of the phases led to the creation of a substantially modified prototype ExoPranayama, which was tested using trials with two small yoga classes (P5). Each of these phases is described in more detail below. During phase 2 to phase 5, we collected audio and video data and conducted a series of interviews. Interviews were fully transcribed and approached with an inductive thematic analysis, with the data iteratively coded at a low level and grouped together to reveal relevant themes.

\section{Phase 1: observing a yoga practice}

While other technologies such as Sonic Cradle were intended to introduce non-practitioners to respiratory interaction [10], our goal is to design technology for those already active in a yoga practice. Thus, prior to iteratively re-designing the technology, we first looked to better appreciate the intricacies of the practice itself. In order to gain a deeper understanding of how yoga is practised and taught, we adopted a participant observation approach, where one researcher in the team took part in a weekly yoga class over a 3-month period. In presenting our

Yoga, meaning "union" or "joining", is the practice of balance and unity of the body and mind [16]. The overarching goal is to reach a sense of union between the self and the world, resulting in inner peace or awareness. This is typically achieved through the practice of a variety of physical and mental exercises. These include a range of physical postures called asanas, such as standing or seated positions. A yoga teacher will give verbal instructions throughout, guiding students' into the asanas limb by limb.

\subsection{The role of breathing in yoga—Pranayama}

Alongside physical movement, awareness and control of the breath are vital aspects of a yoga practice: "breathing to me is absolutely key [...] it was almost a light bulb moment when I started thinking it was as much about the breath as it was about the postures" [T4]. Being conscious of the breath is essential throughout all of the asanas, and in some cases, it is synchronized with physical movement to form vinyasas or yoga flows: "[the breath] is essential [...] it's completely interwoven $[\ldots]$ a lot of the time we will be moving with the breath" [T3]. Purely controlling the breath is also practised in its own right, with a series of dedicated breathing exercises called Pranayama. During yoga classes, only a small segment is devoted to this practice, due to the levels of energy, control and concentration required.

Although yoga is an individual pursuit [6], it is most often taught and practised socially within a group [17]. This social 
aspect is considered to add value to the practice: "I practice on my own regularly, but it's not the same as being in a group and [...] sharing an energy with all those people around you" [T4]. The sense of group cohesion is interestingly attributed to group mindfulness of breath: "there's this state of connectivity that happens when people start breathing at the same rate [...] a connection which I think for me is the underlying fundamental thing that humans look for and people get out of a yoga practice" [T5]. In yoga then, the breath is not only a source of internal awareness and union, but also social awareness [6] and connection [17].

While there are many social benefits to practising yoga in a group, there are also some disadvantages. These come as a consequence of significant physical differences between individual bodies, and gaps in the skill and duration with which yoga students can execute each asana [6]. For this reason, a teacher must carefully consider each student's abilities [17] and "will adjust their class to accommodate that person so that they are just working in the right way for them" [T1]. This was observed throughout the yoga classes, as the sequence of asanas and their difficultly are tailored to the "average level" of the class, with more experienced students kept engaged by individually guiding them into more complex versions of the same asana (teachers must discourage competitiveness due to risk of overexertion [6]). This type of personalized teaching is also applied to Pranayama where skill levels differ: "from what I observe in a class when we do a breathing exercise together, people are mostly in tune, but not quite, because people will have a different kind of capacity" [T5]. It is very common in a yoga class for teachers to remind their students to breathe, as beginners frequently forget because they overly focus on the choreography. Students can even breathe too much: "unless you as a teacher keep a good control of the class, they will all speed up... and they will start getting carried away" [T5] and "people can have real serious panic or anxiety attacks" [T1].

\subsection{Tailoring and guiding through feedback}

As we observed, it is through feedback that teachers personalize their lessons and give guidance to their students. From the student's perspective, they get auditory or physical feedback from their teacher or props. Students can also receive visual feedback, even related to breathing: "In pranayama you can demonstrate, so if you're looking for a viloma breath [note: a simple breathing technique designed for beginners], you can show that on your body, you describe what you're doing, and then get them to look at the movement before they then do it" [T2].

Teachers receive feedback by observing students' physical movements: “you can watch somebody's breath when they are lying down, you can see how they are breathing" [T3] and "you'll see how they are coping, and if they are struggling a little bit then you know that straight away so you're constantly observing your students and seeing how they are reacting to what you are doing [...] that kind of subtle feedback" [T1]. This observational feedback is important in maintaining the safety of students, and teachers will move around the classroom to close inspect their students' well-being. The problem that teachers face is that while they can see whether instructions are being followed, they cannot always accurately tell how well they are being executed: "You can observe to some extent what somebody is doing, and you know whether they are getting the practice, but you don't necessarily know how they are practicing it, and whether they are getting the benefits" [T4]. There is clearly a gap in the information teachers can attain from observing students in the traditional way: "it's really hard to know whether you're breathing correctly [...] you don't have that level of awareness" [T3].

In the light of our observations, it is clear that breathing is an integral aspect of a yoga practice, although with it comes a number of challenges associated with the teaching of groups of varying experience in a controlled way. While there is a series of visual and auditory cues which can help teachers to judge the state of their students during a practice, there appears to be room for deeper insights into the internal state of students, such that teachers may be more effective with their instructions. Even though technologies exist that reveal this type of information (e.g. heart ratetracking wearables www.mioglobal.com or apps www. azumio.com), they do not necessarily complement the practice itself: they require an active form of interaction or present technical information that requires interpretation.

The observations from the yoga classes and limitations of existing technologies appeared to indicate much potential for ExoBuilding and its physical manifestation of breath to support both individual respiratory feedback and group interaction.

\section{Phase 2: engaging with yoga teachers}

For the second phase of the design process, we focused on attaining the views of teachers on the idea of using ExoBuilding in a yoga practice. A 3-h workshop was organized, consisting of in-depth group interviews with teachers about their practices, individual trials of driving (operating) an unmodified ExoBuilding with respiration data and post-use discussions about their experience. Five qualified teachers (4 Female, 1 Male) agreed to participate, they were aged between 30 and 50, and each had at least 5 (and up to 30) years of experience. Participants collectively 
agreed that their style of yoga be anonymized for this research (each was different), as they did not want to be seen as mistakenly endorsing ExoBuilding on behalf of their school of yoga. Participants were given 10 min each to lie down in the environment and try out different breathing exercises. Below, we collate the key results following the teachers' experience of ExoBuilding.

\subsection{Feedback from the environment}

Having practised different aspects of Pranayama within ExoBuilding, participants reflected on the impact of the environments they regularly practice yoga in:

"To have a place you can go, a regular environmental influence on people coming to a space and making that their regular place of practice, it's that kind of thing that allows people to go a little bit deeper into a practice, cause they know it's a safe group and a safe space" [T1]

They likened ExoBuilding to established feedback tools which they already use in their practice: "I have a yoga swing and yoga ropes in my yoga room, it's just another thing you can unhook" [T2], and also how the permanency of the structure can add value, as it: "might encourage what we've lost, which is a consecrated space for practicing yoga in your home" [T1]. In terms of interaction, participants felt ExoBuilding was a unique external experience:

"What's really key to [the breath] for me, without it, you can't really get to a state of pratyahara [...] where the mind is withdrawn from the external experience, so this is quite interesting, especially thinking about [ExoBuilding], how can the context of the external influence the journey inwards?" [T1]

This externalization highlighted the types of information that ExoBuilding can reveal:

"You don't consider what the environment is doing, how you're breathing or what your heart rate might be, so it kind of brings all of those things into your awareness which you're not normally thinking about" [T1]

The group also explored how this biofeedback might work in a group situation. All of the teachers laid down inside ExoBuilding, one teacher drove it with their breath, while another gave the group direct instructions to follow:

"It gave me some feedback to how [T2] was breathing, so I could tell [T2] was able to do [it] because I did a practice where you roll the breath down the length of the body. It gave me some feedback straightway, a lot more than you would usually get, you can't hear someone's breath in that way, you can't see the way someone is breathing" [T1]

For the participant driving ExoBuilding, even such a short experience with feedback indicated positive signs on their practice: "I think I found I got more deeply into pratyahara more quickly" [T2]. However, there were issues for those teachers sharing the space and trying to follow the driver's breathing pattern:

"[T2] might have thought [their] breath was regular, it wasn't. For me it would have to be really steady, I would prefer for that to be controlled. The idea behind yoga practice is all control isn't it, and a human isn't even in the way they breathe, whereas I need it to be consistent" [T3]

\subsection{Bringing biofeedback to yoga}

Yoga is a unique practice as it is made up of both spiritual and physically strenuous activities. Many of the perceived positive by-products of a yoga practice have been scientifically proven to be beneficial [18], and so it is interesting to see the biofeedback create a divide amongst our teachers, with some seeing ExoBuilding adding value:

"It's hard evidence which I love because I'm always trying to bring science into the classes so that we are not so far out there that it's not accessible for people. Don't get me wrong, yoga as we all know is multifaceted, and it has it's philosophical spiritual elements but it has to be grass roots to begin with otherwise you would just lose everyone" [T3]

While others felt the biofeedback might detract from the spiritual practice:

"It takes it into the realm of the scientific, when, obviously traditionally yoga is about a spiritual practice, perhaps connecting with something that's bigger than you but more unknown, it makes it more clinical" [T4]

On further reflection, for some, the use of live physiological data revealed a number of social concerns:

"That kind of feeling of competitiveness, it's about numbers" [T5]

"It's an opportunity for me to get away from technology. It would have to be done in a way that was not intrusive, if it was designed to be part of a yoga environment, but was not looking and feeling like technology" [T4]

Although in general, it was felt that there might be a place for ExoBuilding, depending on the intended outcome of its use: 
"It is a question of evidence vs. experience, what's more important? [...] In an ideal world you'd have the experience and you go 'oh yeah well this is evidenced by...' but one can have an impact on the other" [T4]

\subsection{Summary of phase 1 and 2 findings}

To summarize our finding across phases 1 and 2, the potential of ExoBuilding was viewed positively by many. However, even this early on in the investigation, there were also specific issues which it seemed useful to capture and follow through [19]. The introduction of physiological data, supporting quantification of the practice, had the potential to support quicker attainment of key yoga skills. This faster attainment of skills might be undesired though when full self-awareness is the actual aim, and there is also the risk that the quantification of data will lead to competition between group members. The second issue is related to the introduction of any technology into the practice. Both the necessary procedure to fit physiological monitoring equipment and effects of any slight malfunctions could potentially disrupt the "inner peace" strived for in a yoga session. The final issue relates to a more general reflection about very personal, physiological data becoming publicly visible through ExoBuilding. The required trust between teacher and student and within yoga groups is certainly part of yoga practice as it stands now and sharing difficulties with regard to following the class is also common. Ethical issues clearly arise, when individuals might feel like they have discovered a health issue (e.g. related to their respiration) in public, compounded by the fact that physiological data are not collected in a medical context and no medical expertise to analyse such data on the spot is available. These social considerations provided us with a guide for the following design phases of this work.

\section{Phase 3: a group class}

The next phase of the design process was to explore how ExoBuilding might work with a full yoga class. We recruited a local yoga group and their teacher (T6), who set about designing a "typical" yoga session that incorporated ExoBuilding. Following the feedback from P2 regarding the limitations of using biofeedback in a group, we decided to use ExoBuilding in its machine-driven mode. ExoBuilding was set at an agreed pace of $3 \mathrm{~s}$ in both upand-down motions (using a sine wave). This created a 6-slong "breath" (10 breaths per minute) and was maintained throughout the session, with a request from the teacher that we "kept the rate constant... for simplicity" [T6]. The trial itself was scheduled during the group's usual session, designed to last $40 \mathrm{~min}$ and used familiar music (to act as the same relaxation trigger). Twelve students attended (S1S12), each varying in age and experience. T6 ran the session from within ExoBuilding (see Fig. 3), with the students facing the teacher "in a circle that promotes union" [T6].

\subsection{Alignment with a group practice}

In this trial, ExoBuilding was used in a support role, providing students with a rhythm to follow. For one student, ExoBuilding took on one of the roles of a teacher: "We all sometimes forget to breathe properly, so [ExoBuilding] reminds me to breathe" [S5]. For some students, the sounds from the servomotors were more useful than the visual feedback, as might be expected from a practice, which typically involves audible instructions:

"We tried to mimic the breathing of [ExoBuilding]. I found this easiest when I could listen to the building... it sounded a little like in and out breaths" [S3]
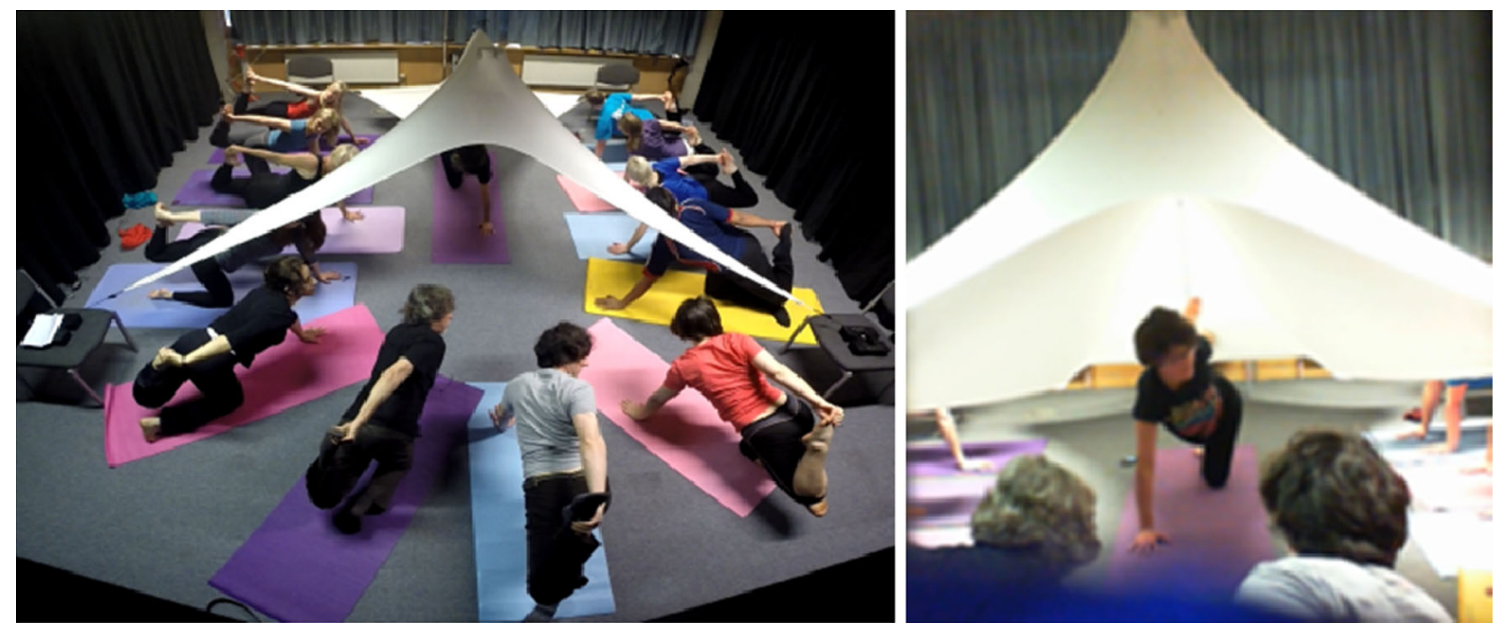

Fig. 3 A large yoga class taught with support from ExoBuilding 
A major question raised by many was the appropriate size for a group using ExoBuilding: "I'm not sure it's suited to a group, or it would have to be a much smaller number" [S6] and that "it would work really well with 3 people" [S3]. A more advanced student doubted ExoBuilding's use in a group practice: "I wonder if it would ever work in a group situation, as breathing in yoga is such an individual experience" [S4].

\subsection{Individual differences in breathing}

It was clear throughout the session that individuals within the group were having quite different experiences in terms of their breathing, with some gaining a positive effect from ExoBuilding, where it "helped me to regulate my breathing" [S2] or gain "a sense of collective and improved breathing" [S5], while others felt "it was too fast" [S7] or "a little off putting if you lose your rhythm" [S4]. This is something we anticipated from our earlier observations of non-ExoBuilding classes and were interested to see how the yoga teacher would approach this challenge:

"The more advanced you are the slower you breathe, so people not used to it will breathe quite fast [...] the difficulty is that not everybody is advanced. So I thought an average is going to be 3 in 3 out" [T6]

Interestingly, while the pace was the problem for the less experienced students, it was the symmetry of the rhythm that was an issue for the more advanced:

"My out breaths are longer than my in, and I sometimes pause between exhale and inhale, so the timing of [ExoBuilding] did not suit me" [S4]

One suggestion was to provide the teacher with some means of controlling the rate of ExoBuilding:

"Whoever is leading the class should have control of the speed" [S1]

Biofeedback was also suggested as a way of creating a more natural breathing rhythm for the group:

"A smaller session with [T6] teaching, and someone else being attached so that the group could try and synchronize with that person. It would make for a more natural breathing rate" [S3]

\subsection{Summary of phase 3 findings}

Summarizing phase 3 of the development process, it was clear that ExoBuilding continued to show promise for yoga practice. With the prototype driven through a regular pattern, its potential to act as a teacher or lead emerged for the first time, while it also seemed too small for the particular group we trialled it with. The regular one-size-fits-all approach also meant that group differences in yoga practice were highlighted with some participants falling in sync with the rhythm, while others did not agree with its pace or the regularity of it. It was suggested that the yoga teacher should have more control over the pace and rhythm of the prototype movement in future iterations.

\section{Phase 4: paired yoga}

Following the group session, we made a number of changes to ExoBuilding, centred on different control configurations. We invited T6 and one of their students (S4), who best matched their skill set to participate. Over a period of 6 weeks, we carried out 3 one-hour trials with the pair, each time introducing new features to ExoBuilding. During the trials, the participants chose their own asanas and breath exercises, as if they were doing their typical practice. They were also instructed to use the features of ExoBuilding as they saw fit. This added to the veracity of the trials, helping to create distance from the feeling of being studied. For the trials, participants each wore a respiration belt. Each trial is detailed below, including the changes introduced (see Figs. 4 and 5):

Trial 1: A control box was created using a Phidget slider sensor (www.phidgets.com), which provided the participants with a local means to adjust the automated motion rate of ExoBuilding (limited to the same rate in both upand-down directions). We then asked them to try 3

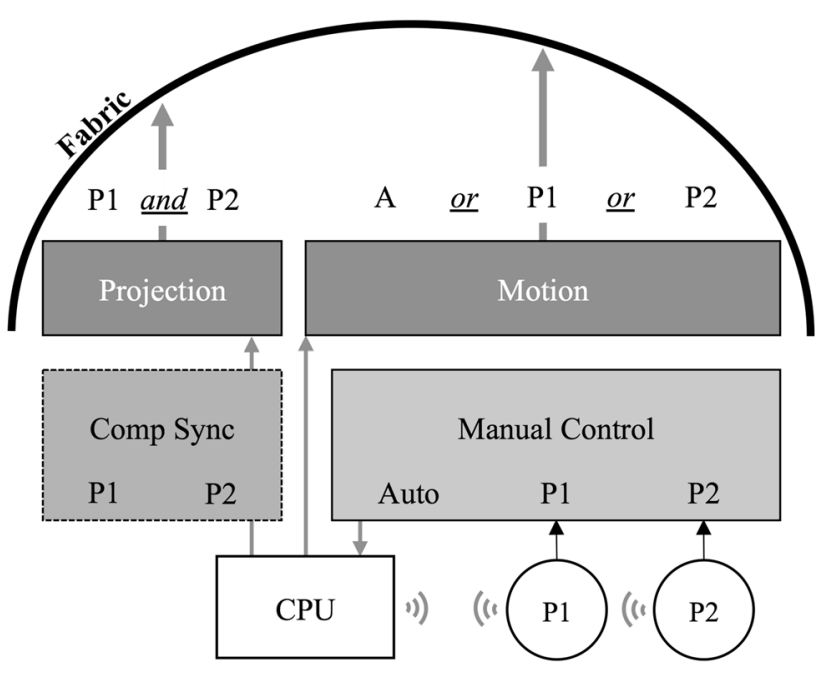

Fig. 4 ExoPranayama-technical diagram of the modified ExoBuilding prototype: participants 1 and 2 have manual control over who/ what drives the movement of ExoPranayama. This can be auto(matic), P1 or P2. The projection of the Om symbol is controlled by the computed level of synchrony between the respiration rates of P1 and $\mathrm{P} 2$ 
Fig. 5 Example features we introduce to ExoBuilding including: a control of rates and operator (Auto, P1, P2), b increased space using poles, and $\mathbf{c}$ biofeedback projected visually as the "Om" symbol, controlled by the level of synchrony between P1 and P2 (regardless of what control was set to)

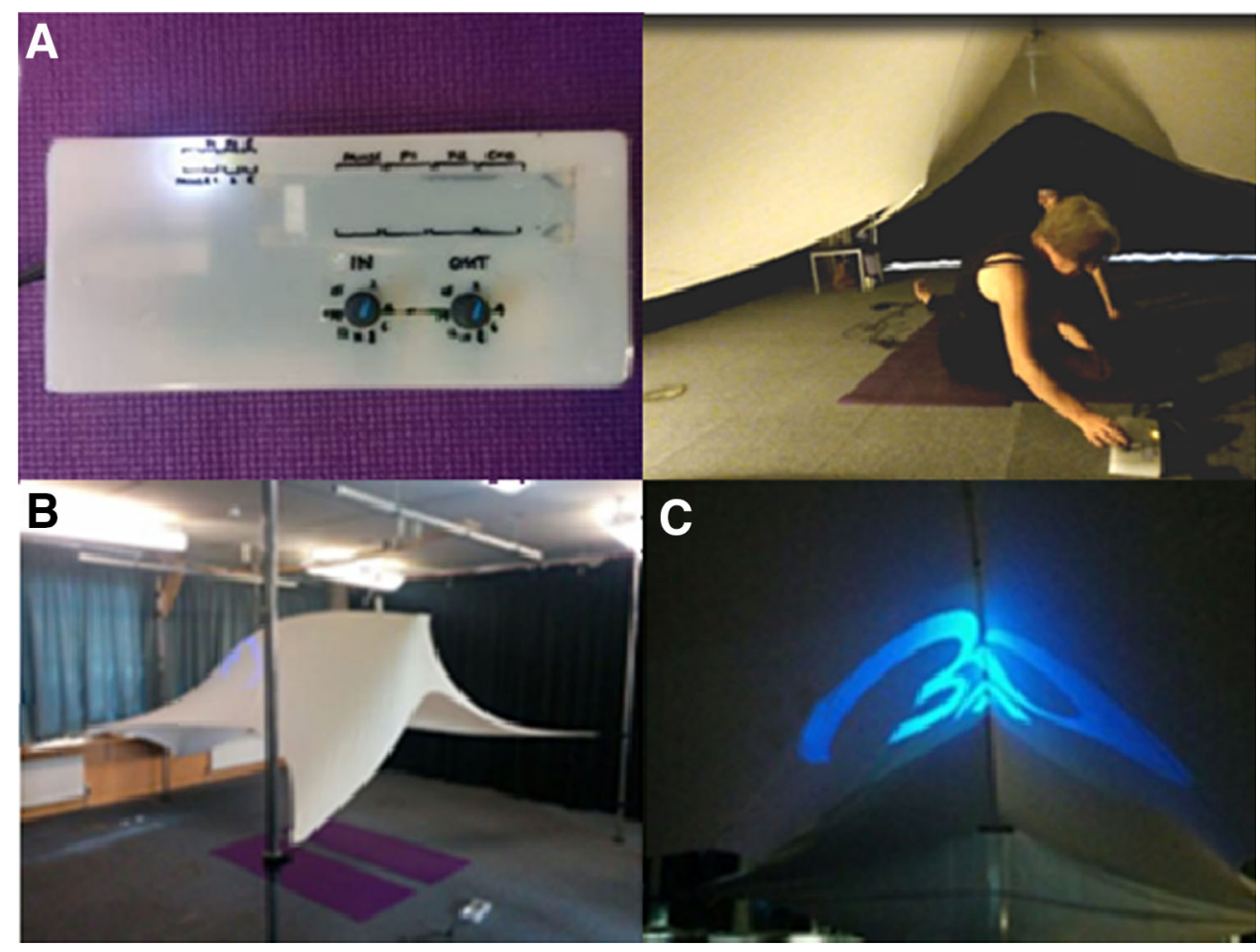

different control configurations: T6 leads, S4 leads and ExoBuilding leads.

Trial 2: We modified the control box to allow the participants to: (1) switch between the three control configurations themselves and (2) adjust the duration of the up-and-down motions of ExoBuilding independently. This involved using additional Phidgets, including a touch slider to emulate select buttons (with corresponding LEDs) and two rotation sensors for adjusting the motion rate of ExoBuilding.

Trial 3: We introduced a projection of ॐ (Om: a symbol of relaxation well known in yoga), on to ExoBuilding, which fades or brightens depending on the degree of synchronization of the breath of the participants with each other. The more synchronized the breaths were, the brighter and more focused the symbol appeared. This was to explore how the two respiration streams could be combined and shared with participants. ExoBuilding was also raised up on poles in order to provide more horizontal and vertical space.

To summarize the interaction enabled through these changes, each participant had their own respiration belt, and the data sets of both were independently streamed in real time to our platform. Only one participant's data would physically actuate the environment at any time. The control box allowed the participants to decide on whose active data were used. In contrast, the projection of the Om symbol was determined by a function of the two data streams. The brightness of the projection would change depending on the synchronicity, Pearson correlation over $8 \mathrm{~s}$, between the two respiration signals (high synchrony: bright, low synchrony: dim/invisible). The key findings across the three trials are presented below.

\subsection{The machine as the master}

When ExoBuilding was used in machine-driven (auto) mode (like in P3), its role became better defined for both participants. ExoBuilding provided a rhythmic pace to follow, and the participants started to talk about ExoBuilding as if it were an instructor/teacher:

"[ExoBuilding] is like a master...yoga master...it makes everything extremely easy, to focus, to concentrate and to improve your practice" [T6]

"It feels more like a trio than a duet, which is nice [...] [ExoBuilding] being the master, a conductor" [S4]

The participants tended to attribute this role to ExoBuilding as they felt it gave a sense of encouragement and coaching:

"I think we were both using that to stretch ourselves a bit. So I can see this as being a really useful teaching aid or learning aid, in order to learn how to deepen and lengthen the breath" [S4] 
In fact, it was suggested that ExoBuilding provides a better rhythmic breathing practice than a human:

"We did try it so I would be talking while [T6] was breathing, and vice versa, but we couldn't really get that right ... so for a good yoga practice, I think it's better to have the building to do the breathing" [S4]

During biofeedback, participants found it difficult to breathe in a predictable way. This caused problems for T6 when trying to pre-empt S4's next inhalation:

"The second delay was a bit... my brain was not liking it $[. .$.$] unfortunately humans are more erratic$ than machines" [T6]

This erratic breathing created a barrier to the group's cohesion. Fortunately this was completely resolved when both followed a machine-driven breath:

"It was great, it was like... the sense of connectivity to yourself and to others that yoga brings union... I felt very well connected to [S4]" [T6]

Providing participants with a means to finely control the duration of the in-and-out motion of ExoBuilding allowed them to find a natural rhythm they could both follow:

"The breathing was too slow for this particular position, so we needed to increase it [...] that worked better because we were able to come to an agreement where we felt the pace of the breath needed to be" [S4]

\subsection{Shared awareness through biofeedback}

While there was a general preference for a machine-driven ExoBuilding amongst the pair, biofeedback also added value to their practice. The movement of ExoBuilding helped provide secondary feedback on participants' strengths and weaknesses:

"With [T6] controlling it, certainly in those backbends, I think [T6 is] stronger than I am, I felt my breath was shorter than [T6's], [T6 was] able to hold those positions for longer [...] I was aware that I'd be struggling to hold that breath, and [T6] was still on the up breath" [S4]

After several trials of ExoBuilding, the participants showed a desire "to have a different kind of feedback [...] would be really interesting" [S4]. It was for this reason the projection of ॐ $(\mathrm{Om})$ was added. This additional feedback was well received and added a new layer of awareness: "The lights are great [...] more feedback, more info, more awareness of how well your breath is synchronized with your partner" [T6].

\subsection{Adapting the practice to the environment}

While we made every attempt to make the environment familiar and comfortable for the participants (same campus, day, time and music), it was inevitable that the new surroundings would influence their yoga practice. In addition to the iterative changes we made to ExoBuilding in response to the participants, the participants in turn started to alter their practice in response to the new technology. Many asanas in yoga involve dynamic full body motions, and due to oversensitivity in the sensors, these motions added interference to the actuations. A consequence of this was that the participants focused on a subset of asanas:

"It's all about adapting the asanas to the building [...] the less dynamic the better, twists were the best, because you are very still" [T6]

This adaptation led to some unexpected positive experiences not normally found in a yoga practice:

"There is something about the way we naturally positioned ourselves. We are going in opposite ways and mirroring each other. That's happened because of the confines of the space that we have to fit in, but actually, it's a very beautiful way to do yoga with another person" [S4]

"It's a way to connect with others at that very intimate level, very spiritual, very therapeutic, very rewarding" [T6]

Unfortunately, the confines of the space also meant that some core aspects of a yoga practice were not possible:

“The way it's configured at the moment, it's more successful when we were doing ground-based work or kneeling, or sitting" [S4]

These limitations led to requests to alter the environment: "maybe a little bit wider... It's not essential that you're absolutely inside it but it certainly helps to feel part of the trio" [S4]. In response, we increased the height and width of the environment, and the floor space it adopts, using a set of poles (see Fig. 5b).

\subsection{Summary of phase 4 findings}

Summarizing phase 4 of the development process, the three trials with our pair of yoga practitioners provided additional insights into the role of ExoBuilding as teacher and master, especially when people had fine-grained control over its regular motion. Biofeedback was valued highly when it provided additional information about the yoga partner but also about the level of synchrony between the two practitioners. And finally, we observed how the pair 
adapted their practice to accommodate the ExoBuilding prototype and how this provided for unexpected value in bringing the two more closely together.

\section{Phase 5: teaching pairs}

Following the design process and a series of iterative development cycles, a new prototype technology had emerged. This new instance of ExoBuilding is called ExoPranayama, which more accurately describes the use of the environment within the context of yoga (compare Fig. 4). With no further iterations to be made to ExoPranayama, the next steps were to test the new features of the environment as a teaching aid. We contacted two teachers who had previously used ExoBuilding (T3 and T6) and asked them to design a 1-h yoga class involving a pair of equally skilled students who had not used ExoBuilding before. Our only request was that they try to use all the features (biofeedback and automation) of ExoPranayama at least once during the lesson. T3 designed a session focused on breath and relaxation exercises and chose to sit outside ExoPranayama while relaying instructions. The students were retirees with known difficulties in regulating their breath (S13, S14). T6 opted for a more asana-focused session but in contrast, sat inside with the students, who were aged between 20 and 40 and were intermediate practitioners (S15, S16). The following describes the key findings from both sessions.

\subsection{Revealing the unexpected through biofeedback}

Both teachers felt that the feedback from ExoPranayama provided them with useful information:

"[S13]'s breath is more noticeable, it's easier to see" [T3]

"Normally you are looking at the belly and chest. Now, the information I receive from [ExoPranayama] is more clear and I don't need to look at anybody" [T6]

This information revealed aspects of the students' practices that the teachers were not aware of, and, in some instances, proved to contradict their thoughts:

"I also noticed with [S14] that on a couple of the postures, even though [they've] got much more even breath normally, it was not as even, so that gave me a bit of uhh I suppose...it surprised me that did" [T3]

"I noticed...I thought both the students would be more similar, but they are not and that was interesting for me to appreciate with [ExoPranayama]" [T6]
This surprised the teachers, who initially felt there was not as much value in the biofeedback as the machine-driven:

"My gut reaction was to try and use the automatic thing as much as possible, but then with [S13] it was fascinating because [their] breathing is quite erratic and quite jagged, and then all of a sudden it went really, steady and I noticed it on [ExoPranayama], so I was really chuffed" [T3]

In response to this revelation, the teachers gave equal weight to biofeedback and machine-driven modes:

"I think there is beauty in both: one is more like where you want to get, and the other one is the current state, and that enhances your yoga experience. Then from a teaching point of view it's knowing where your students are...and how well they are doing" [T6]

\subsection{Teaching with ExoPranayama in practice}

Overall, the teachers saw the added value to their teaching:

"It's all very positive and useful, I suppose it would make me a better teacher as I would be much more aware of people's challenges" [T3]

The teachers used the new information from ExoPranayama to inform the way they taught and led their students in real time:

"I was looking every now and then to see if they were synchronized [...] that gives me a sense of which direction I should be giving next, which instruction should be more focused on explicitly saying 'breathe' or maybe more about the physicality of where they are, point out that something needs correcting [...] it gives you more information about what feedback you should be providing to the students" [T6]

"Much more aware of the instructions I'm giving, I now know about [S13] and [S14], I will address the whole group and say, really pay attention to your breath, really notice that inhale, it would address everybody" [T3]

One suggestion was the use of ExoPranayama for goal setting:

"You can see at which level each student is and how easily they can match their breath to the building, and they can keep lengthening the in and out, it's like assessment level you can aim, you can put targets." [T6]

Although this type of target setting may introduce problems associated with competition and pushing too hard in yoga: 
"You can get a little bit obsessive with your own breath, and when you're not making [ExoPranayama] work the way you want it to" [T3]

"I didn't want them to feel pressure to breathe well because if you focus the mind you can hyperventilate, and for them to feel self-conscious" [T6]

\subsection{Summary of phase 5 findings}

This final phase of the trials using the completed ExoPranayama prototype revealed further details about its potential use in practice. Teachers gave equal value to operating the prototype in biofeedback and in regular modes. The former was seen as useful to understand where students are at with their breathing, while the latter was seen as useful in framing where to get to in practice. Teachers also discussed the value of ExoPranayama for teaching more generally, on the one hand to better understand students' challenges and levels of synchronization but also potentially for goal setting.

\section{Discussion}

We now consider our findings across all phases of the development process together and, in more detail, offer a series of implications for designers of biofeedback technology to support yoga and other physiology-related practices. These considerations are particularly relevant given the current upward trend in adoption of personal biofeedback technologies (quantified-self) [20] and questions surrounding their appropriateness for group use.

\subsection{Bringing real-time biofeedback to a spiritual practice}

Yoga is a unique physical activity in that it brings together aspects of aerobic exercise, meditation and spirituality. Much of the practice is spent mindfully looking inside one's self, exploring the body and the mind. In this sense, yoga can be seen as an internal and interpretive activity, which bridges both body and mind. The main use of biofeedback technology in yoga is to quantify and reveal many of these internal physiological processes to its users to assist their focus and progression. Technology such as ExoPranayama brings to the surface information that is not normally available to practitioners in a unique visual/haptic way. Some of the teachers we interviewed voiced scepticism about the value of this internal information (although this changed after using ExoBuilding). This is a view shared by some yoga technology developers [7], who suggest that physiological data are not needed because yoga, as a practice, already "takes care of this". Our observations of ExoPranayama reveal that there is much to be gained from biofeedback in yoga, particularly given the way it revealed to teachers unexpected truths about their students and created positive connections between students. A similar increase in social awareness was observed in the actuated biofeedback in MoodWings [11], and in terms of yoga, the novel information formed new channels of interaction (i.e. teaching) in the same vein as Social Yoga Mats [6].

Revealing biofeedback information also highlighted an intriguing discrepancy between actual and perceived behaviours. Participants showed a tendency to count their breaths more quickly in their minds compared to reality. Given our internal clocks are linked to our dopamine levels [21], this could be a result of the increase in dopamine from yogic meditative practices [22]. Such a discrepancy also acts as a firm reminder that yoga is a personal endeavour, and interpretation of oneself is an important skill to learn. In fact, interpretation plays a critical role in responding to biofeedback. Consider how users of MoodWings did not know how to enact upon the actuated biofeedback, leading to feelings of discomfort [11]. In ExoPranayama, as the actuation reflects breathing, participants could easily see that in order to slow a rapid actuation, they had to slow their breath. Perhaps this is why we did not observe interpretation-related discomfort.

We did, however, observe a series of tensions that led to discomfort in others when biofeedback was shared. It was difficult and uncomfortable for people to try and follow the breathing of another person (via ExoPranayama) due to a perceived erratic and unpredictable pace. As a consequence, while individual biofeedback supported selfawareness, assisted teaching and improved awareness of others' abilities, it discouraged group cohesion. While there are positive aspects to being aware of others' abilities, there also remains the risk that the information could instil a sense of competition both between students and within oneself. If not carefully considered and managed, the use of this type of quantification could encourage yoga practitioners to unreasonably compare themselves to others and generate unattainable expectations. These group-related findings are particularly noteworthy given the majority of biofeedback actuated systems focus on an individual experience $[10,11]$.

Concerns were also raised about the possible distraction of this new information, as yoga is often used as an escape from technology. This raises a broader question of whether there is generally a place for technology in yoga? Interestingly, throughout the trials, ExoPranayama was not thought of as technology, or even a computing device, and many felt it simply part of the environment, a view that closely matches Weiser's [23] vision of calm computing. 
Implication (1)—Exposure to real-time biofeedback should be carefully balanced against the needs of the practice: the timing, framing and amount of biofeedback should be carefully considered. While biofeedback can bring several benefits to a practice, these could quickly turn into drawbacks if not used appropriately and in respect of individuals. Overexposure to biofeedback might foster competitiveness and draw users' attention too far outward, thus diminishing their mindfulness. With this in mind, one recommendation for minimizing competitiveness when using ExoPranayama might be to limit individual use to experts only or to ensure that a qualified teacher is present during group use. Furthermore, accurately measuring the breath is not necessarily the priority, but instead being mindful of what is comfortable. One solution could be to control the system speed in a fuzzier way using descriptors such as "slow" instead of numbered dials.

\subsection{Supporting a group-based individual practice}

A yoga practice can be carried out in a number of different arrangements: alone, in pairs/small groups or in a large class environment. It is interesting to reflect that yoga, a highly individual and personalized activity, is practised in groups, particularly as these groups more often than not consist of practitioners with varying degrees of experience and skill. This difference in skill led to reservations as to the usefulness of biofeedback in large groups, i.e. if everyone is different, how can biofeedback support a shared experience? From a teaching perspective, the biofeedback helped reveal unexpected aspects of a student's practice and inform the teaching approach. However, difficulty arises when fellow practitioners try to emulate the breathing. Thus, it appears the role and intention of those interpreting the biofeedback impacts their experience of it.

To compensate for the limitation of biofeedback in pairs, we sought to explore the impact of the machinedriven mode. This provided a motion that is not only predictable and easy to follow, but customizable in real time. This led to significant increases in levels of connectedness within the group of users and also interestingly with ExoPranayama itself. Participants described their experience as a "trio", assigning a role to ExoPranayama in the form of a conductor. In this way, ExoPranayama began to emulate aspects of a teacher in guiding the rate of breathing, helping participants push themselves further than they would normally. The limitation is that to achieve such effects, participants need to be of a similar skill level, or else there is a risk of overexertion or reduced engagement.

The feedback in the form of the visual Om symbol (Fig. 5c) provided an additional goal for participants.
Actively seeking a shared breathing pattern in this visual way clearly contributed to the stronger sense of connectedness. In our experience, such abstract representation of shared behaviours can therefore work well, while other representations, for example, through sound have not been explored in our work.

Two key ways of using ExoPranayama in a yoga practice now begin to emerge focusing on current and future states. Firstly, biofeedback can be used to inform about the current internal state of a practitioner. It reveals to individuals, teachers and members of a group the inner workings of one specific person or the synchronicity between two people, which can help inform their development in the practice. The second use of ExoPranayama is in machine-driven mode, allowing groups of similarly skilled practitioners to assign goals (their future state), which they are encouraged to attain. When both practitioners match their breathing to the agreed-upon automated motion of the environment, it can also be used to create a strong cohesiveness within the group, more easily achieving a similar state of connectedness compared to typical classes.

Implication (2)—depending on the intended goal of the practice, feedback should be aligned with the practitioner(s) interactional arrangement: the feedback mechanisms explored in ExoPranayama were found to affect different aspects of the practice. For example, group cohesion was promoted by ExoPranayama's machine-driven state, but reduced when using biofeedback. In contrast, individual awareness was gained via biofeedback, but lost when machine-driven. This compromise was alleviated when using the visual aggregated biofeedback. Hence, the ability to change between different modes of feedback is important given the intended goal and role frequently change within a practice.

\subsection{A flexible practice in response to the space}

Yoga focuses heavily on the principle of looking inwards and to sense both body and mind. ExoPranayama, in biofeedback mode, acts as a physical manifestation of those internal processes and draws the attention of practitioners outwards into the space in order to more deeply look inwards. Incorporating this type of interaction actually started to influence the practice itself. In terms of asana, the more static postures generated the most regular breathing and as such became a focus point for those practising in ExoPranayama. The feedback was so compelling that we observed a tendency for participants to hold the postures for longer compared to ordinary classes.

The physical constraints of ExoPranayama, the height and width, created an added level of restriction on the executable/possible types of asanas. This contrasts with other yoga technologies which deliberately imposed 
constraints (restricted number of supported asanas) on the practice as a consequence of the limitations of their technologies [5, 7]. Interestingly, in the case of ExoPranayama, the constraints led to a number of unexpected positive reactions. The physical restrictions of the environment forced participants to be creative in the dynamics of asanas, modifying the postures in response to the space. In some instances, this led to participants mirroring each other and practising in close proximity, which contributed to the sense of connectedness.

Implication (3): a technology-augmented environment can not only support yoga in new ways, but also facilitate creativity and growth of the core practice: In addition to the biofeedback itself, the means through which it was delivered had an impact on the yoga practice. The constraints of the environment promoted innovative asanas, which would otherwise not have surfaced. This exposes an intriguing interplay between the technology and the practice, where compromise and balance were achieved through (un)intentional iterative changes to both. In some ways, a new style of practice has emerged through the use of ExoPranayama. Other technologies were not as easily adaptable, most likely because they only work with a subset of asanas. ExoPranayama on the other hand focuses on breathing, which permeates throughout an entire practice and across all asanas. Hence, technology developers should focus on supporting the wider processes involved in the practice, as opposed to more specific, and therefore limiting, elements.

\subsection{Design space for technology mediated yoga}

The design space for yoga can be considered in terms of feedback provided (biofeedback or not) and types of user (individual or group) (see Table 1). Most of the existing technologies shown in Table 1 were designed to focus on individual experiences, in favour of group experiences. This reflects a focus on "yoga-in-the-home", with the intention of replacing the yoga teacher and the simplicity

Table 1 Yoga technology design space (\# users vs. technology)

\begin{tabular}{|c|c|c|}
\hline & Non-Biofeedback & Biofeedback \\
\hline 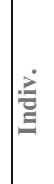 & $\begin{array}{c}\text { Wii Fit }[4], \text { TIYS [7], } \\
\text { Kinect }[5] \& \\
\text { ExoPranayama }\end{array}$ & $\begin{array}{c}\text { MoodWings [11], ExoBuilding [2], } \\
\text { Meditation Chamber [9], Sonic } \\
\text { Cradle [10], i-m-Breath [8] \& } \\
\text { ExoPranayama }\end{array}$ \\
\hline 言 & $\begin{array}{c}\text { Social Yoga Mats [6], } \\
\text { IYS [17] \& } \\
\text { ExoPranayama }\end{array}$ & ExoPranayama \\
\hline
\end{tabular}

compared to multi-user systems. However, yoga is frequently practised socially, and our aim is to cover the whole design space. In fact, the different aspects of the table capture the interactional arrangements possible using ExoPranayama, each with their own effects and limitations (where non-biofeedback includes ExoPranayama in machine-driven mode):

Individual Non-Biofeedback (IN): Increases in motivation to attain specific goal (i.e. set breathing pattern).

Group Non-Biofeedback $(G N)$ : Increase in group cohesion and goal attainment, but only if equally skilled.

Individual Biofeedback (IB): Increases in selfawareness.

Group Biofeedback $(G B)$ : Increases in self and group awareness, but only creates cohesion if data are aggregated (Table 1).

With a better understanding of the types of experience ExoPranayama can bring to a yoga practice, one can begin to see how they could all be used within a single practice. For example, a student could start with IN to relax by achieving a specific state of breathing. Next a teacher could observe the state of the student in IB, adjusting their direction accordingly. Then GB or GN could be used to achieve a sense of connection across a group of students. Finally, IN could be used by the student at the end of the session during shavasana (lying relaxation) to focus on their breathing.

\subsection{Breathing life into HCI}

While this work directly contributes to the wider understanding of physiology as an interactive experience, it also shifts away from the quantified-self movement by collecting group data and taking a more holistic view of the body with links to spirituality through yoga. Techno-spirituality (TS) is a field which explores how technology can support spirituality, and has been shown to be a neglected area of HCI, as Bell discusses in more detail [3]. There are two main classes of system within TS that ExoPranayama relates to: Practical (indirect) and Experiential (direct) enablers of spirituality [24]. In terms of practical facilitation, the machine-driven mode of ExoPranayama offers users a means to tailor their own goals, reaching specific cognitive states by adjusting their breathing rate to ExoPranayama. This idea of looking to an automated environment for behavioural cues, and "submission to external processes", is shared with home automation technologies used during the Jewish Sabbath [25]. For example, the way lights (actuation) turning off dictate time to sleep (behaviour) is analogous to the automated rate of ExoPranayama (actuation) dictating a breathing rate (behaviour).

ExoPranayama also offers an experiential means of fostering spirituality through physical actuations in 
response to biofeedback, similar to Sonic Cradle [10], enabling users to reach pratyahara (withdrawal from oneself) more quickly. Unlike Sonic Cradle, ExoPranayama can support groups of users, creating increases in both awareness and cohesion, two traits potentially beneficial to a group spiritual practice. ExoPranayama also offers a unique means of switching between practical and experiential enablers, by simply changing the driver.

\section{Conclusions}

We have presented the development of ExoPranayama, an actuated biofeedback environment designed to support a yoga practice. The iterative design process saw a total of 22 yoga students, and teachers use the environment at different stages of development. As a consequence of the ongoing observations, we added a series of features to the environment related to local control, visual feedback and physical space. Across the trials, our qualitative results revealed a number of tensions in bringing biofeedback to a spiritual practice and providing feedback to individuals within a group. Through these tensions, two key ways of how ExoPranayama can support a yoga practice emerged: (1) biofeedback can provide new and useful information to teachers about the current internal state of their students, and (2) when a machine drives the environment in a regular and predictable pattern, self-reported group cohesion improves dramatically. Our results demonstrate the applicability of actuated biofeedback environments in yoga, but also heed caution regarding the prominence of their role within physiology-related practices.

\section{Data accessibility}

The analysis of the use of the ExoPranayama prototype draws on observational fieldwork, trials that were audio and video recorded as well as audio-recorded interviews, which could all identify individuals. Participants did not give consent for these data to be published in a publicly available data repository in its raw form.

Acknowledgments We would like to express our gratitude to Nottinghamshire-based yoga teachers Elvira Perez-Vallejos, Jay Rossi and Jeanette Bunyan for their expert advice and all of our yoga practitioners, in particular Liz Lesquereux, for taking part in this research. It should be noted that we do not consider participation in this research as an endorsement of our technology, nor do we advocate any one particular approach to yoga. This work was supported by EPSRC Grants EP/I011587/1, EP/M000877/1, and EP/P505658/1 and the University of Nottingham via the Nottingham Research Fellowship 'The Built Environment as the Interface to Personal Data'.
Open Access This article is distributed under the terms of the Creative Commons Attribution 4.0 International License (http://crea tivecommons.org/licenses/by/4.0/), which permits unrestricted use, distribution, and reproduction in any medium, provided you give appropriate credit to the original author(s) and the source, provide a link to the Creative Commons license, and indicate if changes were made.

\section{References}

1. Al Osman H, Eid M, Saddik A (2013) U-biofeedback: a multimedia-based reference model for ubiquitous biofeedback systems Multimed Tools Appl 1-26

2. Schnädelbach H, Irune A, Kirk D et al (2012) ExoBuilding: physiologically driven adaptive architecture. TOCHI 19:25

3. Bell G (2006) No more SMS from Jesus: ubicomp, religion and techno-spiritual practices. In: UbiComp'06, pp 141-158

4. Esculier J, Vaudrin P et al (2012) Beriault, Home-based balance training programme using wii fit with balance board for parkinson's disease: a pilot study. J Rehabil Med 44:144-150

5. Rector JA, Bennett K, Kientz CL (2013) Eyes-free yoga: an exergame using depth cameras for blind \& low vision exercise. In: ASSETS'13, ACM, New York, p 12

6. Nagargoje T, Maybach A, Sokoler K (2012) Social yoga mats: designing for exercising/socializing synergy. In: TEI'12, ACM, New York, pp 87-90

7. Fels P, Gauthier S, Smith J (2002) Responses in light, sound and scent: a therapeutic interactive yoga system. In: IWEC'02, pp 355-362

8. Yu M, Chen J Chang K et al. (2011) i- m- Breath: the effect of multimedia biofeedback on learning abdominal breath. Adv Multimed Model 548-558

9. Shaw C, Gromala D, Song M (2011) The meditation chamber: towards self-modulation. In: Metaplasticity virtual worlds aesthetics and semantic concepts, pp 121-133

10. Vidyarthi J, Riecke BE (2013) Mediated meditation: cultivating mindfulness with sonic cradle. In: CHI' 13 extended abstracts, pp 2305-2314

11. MacLean D, Roseway A, Czerwinski M (2013) MoodWings: a wearable biofeedback device for real-time stress intervention. In: PETRA'13 2013

12. Reiner R (2008) Integrating a portable biofeedback device into clinical practice for patients with anxiety disorders: results of a pilot study. Appl Psychophysiol Biofeedback. 33:55-61

13. Marshall JD et al. (2011) Breath control of amusement rides. In: CHI'11, 2011, pp 73-82

14. Rennick-Egglestone S, Humble J, Greenhalgh C et al. (2006) The equator component toolkit: managing digital information flow in the home. In: UIST'06, 2006

15. Vredenburg K, Mao J et al. (2002) A survey of user-centered design practice In: CHI'02, 2002, 471-478

16. Larssen A, Robertson T, Edwards J (2007) The feel dimension of technology interaction: exploring tangibles through movement and touch. In: TEI'07, 2007, pp. 271-278

17. Park M, Pinto M, Chen $M$ et al. (2012) Enhancing the experience of yoga practice through an immersive interactive studio. In: APCHI' 12, 2012

18. Varambally S, Gangadhar BN (2012) Yoga: a spiritual practice with therapeutic value in psychiatry. Asian J Psychiatr 5:186-189

19. Jäger N, Moran S, Schnädelbach H (2014) Using adaptive architecture to support yoga practices: social considerations for design. In: Third IEEE workshop on the social implications of pervasive computing for sustainable living, 2014, pp 364-369 
20. Gomez R, Harrison A (2014) Beyond wearables: experiences and trends in design of portable medical devices. In: Design, user experience, and usability. User experience design practice, pp 261-272

21. Meck W (1996) Neuropharmacology of timing and time perception. Cogn Brain Res 3:227-242

22. Kjaer TW, Bertelsen $C$ et al (2002) Increased dopamine tone during meditation-induced change of consciousness. Cogn Brain Res 13:255-259
23. Weiser M, Brown JS (1996) Designing calm technology. PowerGrid J 1:1-17

24. Buie E, Blythe M (2013) Spirituality: there's an app for that!(but not a lot of research). In: CHI'13 Extended Abstract, 2013, pp 2315-2324

25. Woodruff B, Augustin A, Foucault S (2007) Sabbath day home automation: it's like mixing technology and religion. In: CHI'07, 2007, pp 527-536 